\title{
Load Monitoring Based on the Auxiliary Particle Filter Algorithm
}

\author{
Wang Shen ${ }^{1, \mathrm{a}}$, Wei Zhi-qiang ${ }^{1, \mathrm{~b}}$, Yin Bo ${ }^{1^{*}, \mathrm{c}}$ \\ ${ }^{1}$ Ocean University of China, No.238, Songling Road, Laoshan District, Qingdao City, Shandong \\ Province, China \\ àwangshenouc@sina.cn, ${ }^{\mathrm{b}}$ weizhiqiang@ouc.edu.cn, ${ }^{\mathrm{c}}$ ybfirst@126.com
}

Keywords: Non-intrusive load monitoring, Household and appliance model, Particle filter, Bayesian estimation, Auxiliary particle filter, MATLAB simulation

\begin{abstract}
This paper introduces family load monitoring based on auxiliary particle filter algorithm. It mainly uses a set of random samples with relevant weights to estimate the posterior probability density $p\left(x_{t} \mid Y^{t}\right)$. First of all, the model of household electrical appliances is established in this paper, then using the particle filter algorithm to estimate the state. It mainly consists of two parts: including Bayesian estimation and auxiliary particle filter-based load monitoring. Finally, the data collected by the sensor is simulated on the MATLAB platform, and the simulation results are obtained by using the evolutionary auxiliary particle filter algorithm.
\end{abstract}

\section{Introduction}

Non-intrusive load monitoring (NILM) was proposed by Hart in the 1980s [1]. Which decomposes the total load information into electrical equipment information, and then obtains the energy consumption situation and the electricity consumption law of the users. These electricity information has a high application value. It can provide benefits for Power Grid Corp, users and so on [2]. The advantage of the method is that the monitoring data is accurate and reliable. The disadvantage is that the practical operability is poor, the implementation cost is high and the user acceptance is low $[1,3]$.

Compared with the intrusion load monitoring, NILM' s economic investment is small [3,9]. In recent years, with the rapid development of smart home and smart grid, NILM research boom in the world once again rise. However, the research on non-intrusive load monitoring and dis-aggregation (NILMD) is not enough in China. The research on non-intrusive load monitoring and decomposition algorithm is less. Therefore, the particle filter algorithm has gradually become a hot spot in the field of science.

In recent years, many new methods of nonlinear filtering have been proposed in signal transmission and compression, financial data analysis, image processing, load monitoring and decomposition. All of these algorithms are based on the sequential importance sampling (SIS) filter of Bayesian sampling estimation. However, the above studies have not been able to solve the problem of particle number scarcity and computational constraints, so it has not attracted people's attention. Until 1993, Gordon [4] proposed a new SIS-based Bootstrap nonlinear filtering method, which laid the foundation of particle filter algorithm. The key idea of particle filter algorithm is to use a set of random samples with weights, and estimate the posterior probability density based on these samples. When the number of samples is very large, the probability estimation will be equal to 
the posterior probability density. This method is an unsupervised classification method which is suitable for nonlinear and non Gauss interference problems.

\section{Household Appliances Model}

The load of a household is characterized by the power distribution of each household appliance in a household. Therefore, the sum of each appliance power distribution is the total power load, where each appliance model is modeled by HMM [10]. HMM is a hidden Markov model. It describes the state is hidden, a hidden Markov model by a probability distribution function. In detail, an HMM model has $\mathrm{n}$ hidden states, $S=\left\{s_{1}, S_{2}, S_{3}, \ldots, S_{n}\right\}$, As time goes on, the system is transferred from one state to another.Setting: $x_{t+1}$ represents the state of time $\mathrm{t}+1$ and the electrical power consumption of time $t+1$. The state probability of the system at time $t+1$ depends on the state of times $1,2, \ldots, t$, the probability is:

$$
P\left(x_{t+1}=S_{j} \mid x_{t}=S_{i}, x_{t-1}=S_{k} \ldots \ldots\right)
$$

If the state of the system at time $t+1$ is only related to its state at time $t$, the system constitutes a discrete first-order Markov chain:

$$
P\left(x_{t+1}=S_{j} \mid x_{t}=S_{i}, x_{t-1}=S_{k, \ldots . .}\right)=P\left(x_{t+1}=S_{j} \mid x_{t}=S_{i}\right)
$$

If you only consider independent random process at time $t+1$, then the probability can be:

$$
P\left(x_{t+1}=S_{j} \mid x_{t}=S_{i}\right)=a_{i j}, 1<=i, j<=n
$$

Among them, the state transition probability must be satisfied. As shown in Eq.4:

$$
a_{i j}>=0 ; \sum_{j=0}^{n} a_{i j}=1 \text {. }
$$

We get the state transition matrix $\mathrm{A}$, it represents the transition from the A state to the B state. As shown in Eq.5:

$$
A=\left\{a_{i j}, 1<=i, j<=n\right\}
$$

It is also necessary to define an emission matrix B for HMM. In the Household and appliance model, the emission matrix B represents a possible power value for each state of an appliance.

Initial probability:

$$
\pi=p\left(x_{1}=S_{i}\right)
$$

It must also be defined for the HMM.

\section{State Estimation}

\subsection{Bayesian Estimation.}

Various appliances and their environment consisting of system, according to the initial state probability distribution: $p\left(\mathbf{x}_{0}\right)$ and observed data: $Y^{t}=\left\{y_{t} \mid t=0,1, \cdots, t\right\}$. Estimate the current state of the system $\mathbf{x}_{t}$. From a statistical point of view. The estimation of $\mathbf{x}_{t}$ is a Bayesian filtering problem, it can be achieved by estimating the posterior density distribution $p\left(\mathbf{x}_{t} \mid Y^{t}\right)$. 
According to the Markov hypothesis and the Bayes rule, $p\left(\mathbf{x}_{t} \mid Y^{t}\right)$ can be solved by the following two steps:

1) Prediction stage:The model is used to predict the state of the system at the next moment, That is, the system prior probability density $p\left(\mathbf{x}_{t} \mid Y^{t-1}\right)$ is calculated by the Eq.7:

$$
p\left(\mathbf{x}_{t} \mid Y^{t-1}\right)=\int p\left(\mathbf{x}_{t} \mid \mathbf{x}_{t-1}, \mathbf{u}_{t-1}\right) p\left(\mathbf{x}_{t-1} \mid Y^{t-1}\right) d \mathbf{x}_{t-1}
$$

among them $p\left(\mathbf{x}_{t} \mid \mathbf{x}_{t-1}, \mathbf{u}_{t-1}\right)$ is the state transitive density of the system.

2) Update stage:The observing model is used to update the state of the system by observing information, That is, the posterior density $p\left(\mathbf{x}_{t} \mid Y^{t}\right)$ of the system is calculated by Eq.8:

$$
p\left(\mathbf{x}_{t} \mid Y^{t}\right)=\frac{p\left(y_{t} \mid \mathbf{x}_{t}\right) p\left(\mathbf{x}_{t} \mid Y^{t-1}\right)}{p\left(y_{t \mid} \mid Y^{t-1}\right)}
$$

Where $p\left(y_{t} \mid \mathbf{x}_{t}\right)$ is the observed model of the system(observed density). $p\left(y_{t} \mid Y^{t-1}\right)$ is a constant. According to the Markov assumption, under the premise that the current state is known, past measurements and current measurements are independent of each other.

Although the above two steps give an effective recursive strategy for the load decomposition problem, however, in order to obtain the analytical solution of the posterior density $p\left(\mathbf{x}_{t} \mid Y^{t}\right)$ of the system. The integral in Eq.7 must be calculated. This is difficult to achieve in a highly nonlinear load-shedding problem. In this case, an approximate method, such as a particle filter algorithm, can be used to solve the problem [11].

\subsection{The Particle Filter.}

As shown in the previous section, home load monitoring is a Bayesian filtering problem, which amounts to estimating the state of the load at current time-step $t$, given knowledge about the initial state and all measurements $y_{1} \cdots y_{t}$ up to the current time. When the transition and the observation model are nonlinear and/or non-Gaussian, this problem cannot be analytically solved. While particle filter provides an efficient means to tackle this problem.

\subsubsection{The Generic Particle Filter.}

Particle filter is an attractive simulation-based approach to the problem of computing intractable posterior distributions in Bayesian filtering. The idea is to approximate the continuous posterior density $p\left(x_{t} / y_{t}\right)$ in each time step $t$ by a random sample of $i=1,---$, I particles $x_{t}^{i}$ with corresponding probability masses, or weights $\omega_{t}^{i}$. The posterior is then given by the empirical estimate:

$$
p\left(x_{t} / y_{t}\right)=\sum_{i=1}^{I} \omega_{t}^{i} \delta\left(x_{t}-x_{t}^{i}\right)
$$

Where $\delta\left(x_{t}-x_{t}^{i}\right)$ is a delta function centered on the particle $x_{t}^{i}$. Using Eq.9, the integration for computing the prior in Eq. 8 is now replaced by the much easier summation:

$$
p\left(x_{t+1}\right)=\sum_{i=1}^{I} \omega_{t}^{i} P\left(x_{t+1} / x_{t}^{i}\right)
$$

While, since all integrals are replaced by sums and the continuous densities by discrete ones, the 
require normalization step of the filtered posterior:

$$
p\left(x_{t+1} / y_{t+1}\right) \propto p\left(y_{t+1} / x_{t+1}\right) \sum_{i=1}^{I} \omega_{t}^{i} p\left(x_{t+1} / x_{t}^{i}\right)
$$

Is trivial, namely, a normalization of the discrete masses to unit sum.

Assuming a set of particles that approximate the posterior density $p\left(x_{t} / y_{t}\right)$ at time $t$ sufficiently good, the problem is how to sample a set of particles from the new posterior $p\left(x_{t+1} / y_{t+1}\right)$ in Eq.11. Efficiently sampling from the posterior is the central theme of most methods in particle filters literature [7]. The frequently used sampling method is the Sampling/Importance Resampling (SIR) $[5,8]$. However, the SIR particle filter requires very many particles for convergence when the likelihood function $P(y / x)$ is too peaked or is situated in one of the prior's tails [6]. As a result, the posterior cannot be represented with reasonable accuracy.

\subsubsection{The Auxiliary Particle Filter.}

An elegant solution to the problem of optimal sampling from the posterior has been given by Pitt and Shephard [6] under the name'auxiliary particle filter'. Their algorithm comes down to the following; in order to sample from the posterior $p\left(x_{t+1} / y_{t+1}\right)$ in Eq.11, just insert the likelihood inside the prior in Eq.10.

$$
p\left(x_{t+1} / y_{t+1}\right) \propto \sum_{i=1}^{I} \omega_{t}^{i} p\left(y_{t+1} / x_{t+1}\right) p\left(x_{t+1} / x_{t}^{i}\right)
$$

And treat the products $\omega_{t}^{i} p\left(y_{t+1} / x_{t+1}\right)$ as the component probabilities in order to sample from the respective $p\left(x_{t+1} / x_{t}^{i}\right)$. Because the likelihood $p\left(y_{t+1} / x_{t+1}\right)$ in the above product involves the unobserved state vector $x_{t+1}$, an approximation of Eq.10 has been suggested in [8] as:

$$
\hat{p}\left(x_{t+1} / y_{t+1}\right) \propto \sum_{i=1}^{I} \omega_{t}^{i} p\left(y_{t+1} / \mu_{t+1}^{i}\right) p\left(x_{t+1} / x_{t}^{i}\right)
$$

where $\mu_{t+1}^{i}$ is any likely value associated with the $i$-th component transition density $p\left(x_{t+1} / x_{t}^{i}\right)$, for example its mean.After a set of particles $x_{t+1}^{j}$ have been sampled from the transition density $p\left(x_{t+1} / x_{t}^{i}\right)$ with probability $\omega_{t}^{i} p\left(y_{t+1} / \mu_{t+1}^{i}\right), j=1, \cdots, I$, their weights are set proportional to:

$$
\omega_{t+1}^{i} \propto \frac{p\left(y_{t+1} / x_{t+1}^{j}\right)}{p\left(y_{t+1} / \mu_{t+1}^{i}\right)}
$$

where $\mu_{t+1}^{i_{j}}$ is the associated likely value of the component $p\left(x_{t+1} / x_{t}^{i_{j}}\right)$ in Eq.13 from which the particle $j$ was sampled.

The auxiliary particle filter can be regarded as a one-step look-ahead procedure, where a particle $x_{t}^{i}$ is propagated to $\mu_{t}^{i}$ in the next time step in order to assist the sampling from the posterior. The resulting method is particularly efficient,since it requires only the ability to sample from the transition model and evaluate the likelihood function $p\left(y_{t} / x_{t}\right)$. This makes it very attractive compared to alternative methods that require specialized data structures for sampling from the posterior. However, in the problem of family load monitoring, the auxiliary particle filter will also need a large number of particles to represent the PDF and suffer from the sample impoverishment problem to some degree. 


\section{Experimental Process and Analysis}

We can use the HMM model to synthesize the power load of the family one day, and the results are shown below Fig.1.

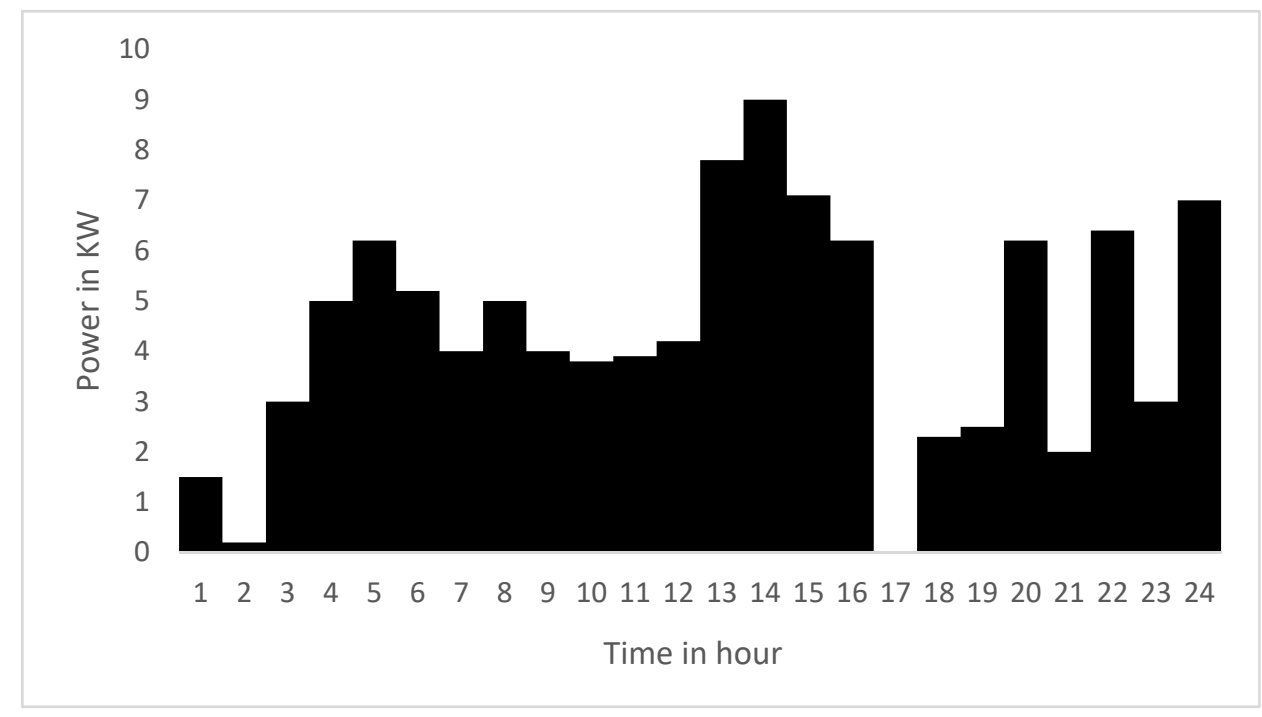

Fig.1.Household power load by synthetic data generation

In this paper, we study the typical household appliances as the research object, the object of this study are: air conditioning, electric light, electric fan, computer, electric kettle.

Using particle filter algorithm in the MATLAB simulation, through the voltage sensor and current sensor to detect the voltage and current sampling data, the electric light and electric kettle of the transient waveform and the five kinds of electrical appliances to open and close the results of the event.
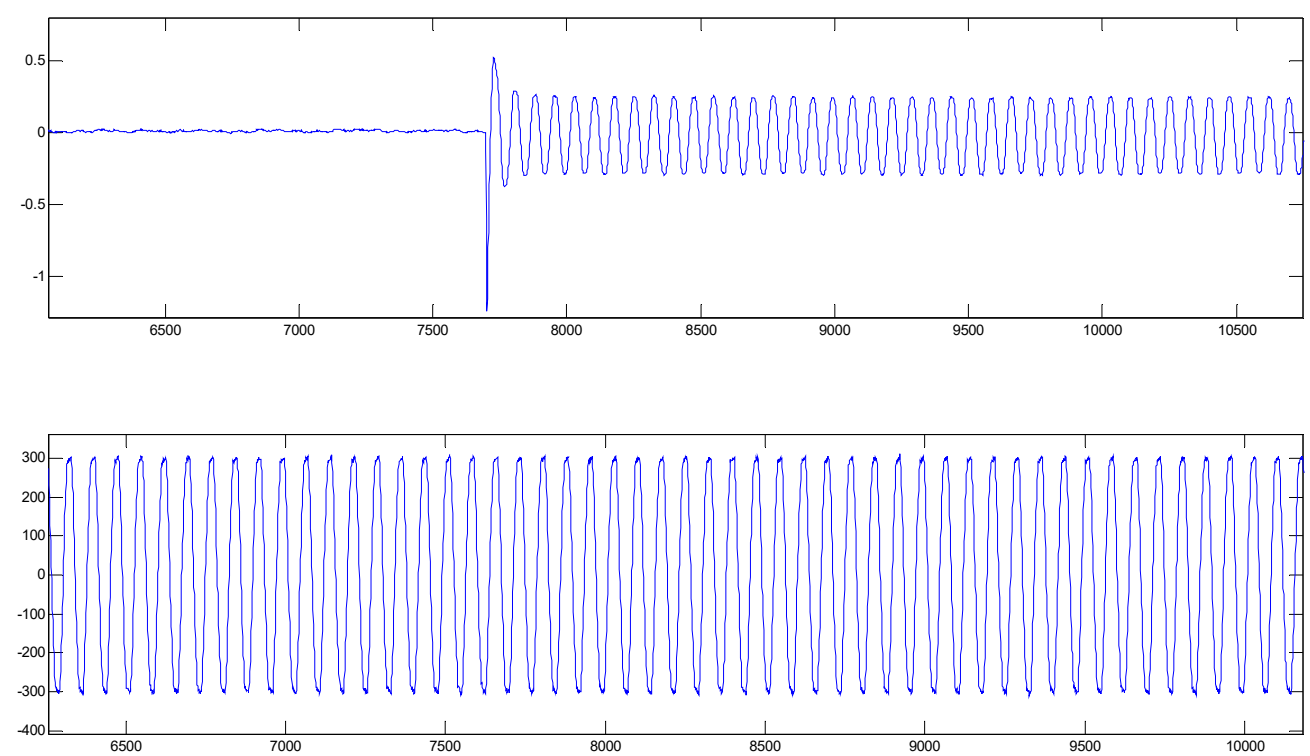

Fig.2.The current and voltage of electric light in MATLAB drawing

The Fig. 2 above is the current map, the horizontal coordinate is the data point sequence, the vertical coordinate is the current (A), the picture below is the voltage diagram, the horizontal coordinate is the data point sequence, the vertical coordinate is the voltage $(\mathrm{V})$. 

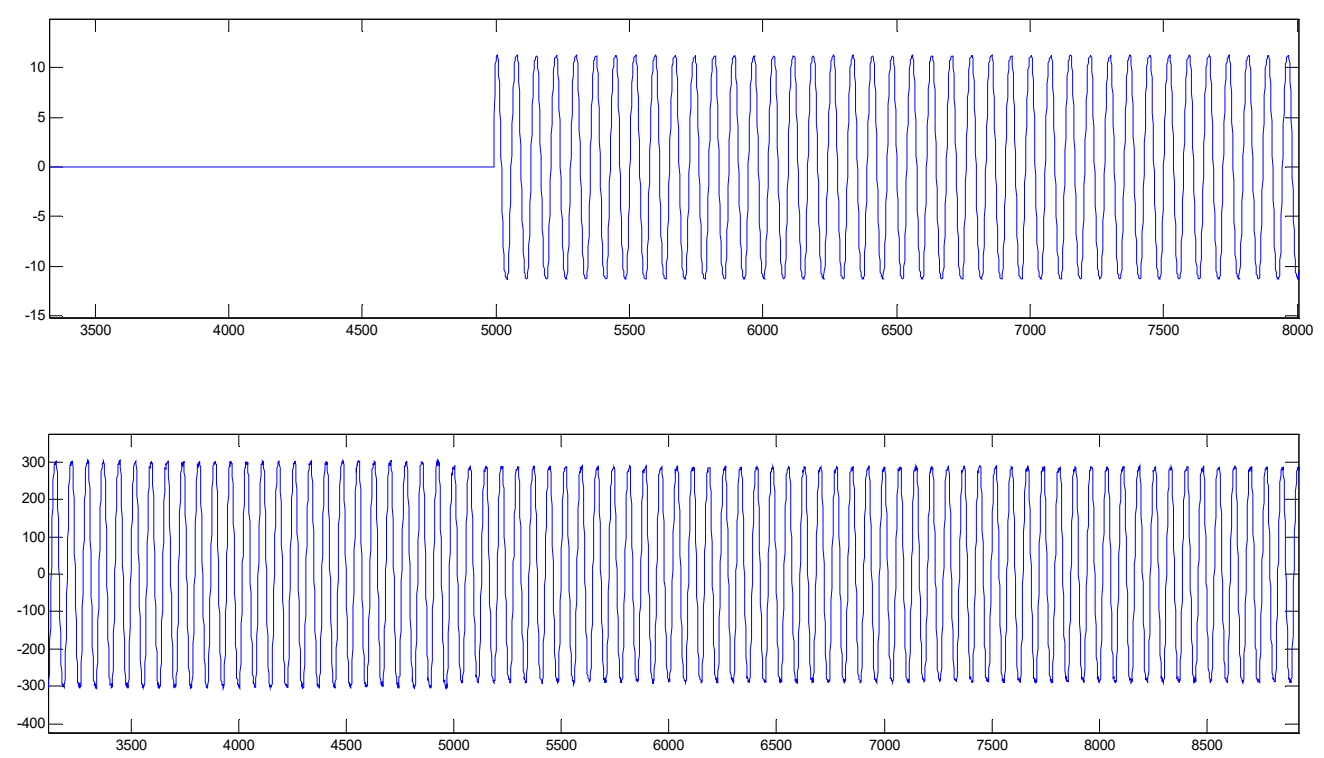

Fig.3.The current and voltage of electric kettle in MATLAB drawing

The Fig.3 above is the current map, the horizontal coordinate is the data point sequence, the vertical coordinate is the current (A), the picture below is the voltage diagram, the horizontal coordinate is the data point sequence, the vertical coordinate is the voltage $(\mathrm{V})$.

The following Table1 is the result of the opening and closing events of the five kinds of electrical appliances.

Table1.the result of the opening and closing events of the five kinds of electrical appliances

\begin{tabular}{|c|c|c|c|c|}
\hline \multirow{2}{*}{$\begin{array}{c}\text { Electrical } \\
\text { appliances category }\end{array}$} & \multirow{2}{*}{$\begin{array}{c}\text { Opening } \\
\text { times }\end{array}$} & \multirow{2}{*}{ Off times } & \multicolumn{2}{|c|}{ probability } \\
\cline { 3 - 5 } & & & $N_{p}=100$ & $N_{p}=1000$ \\
\hline computer & 50 & 50 & 0.9770 & 0.9846 \\
\hline Electric light & 50 & 50 & 0.9807 & 0.9898 \\
\hline Electric fan & 50 & 50 & 0.9744 & 0.9901 \\
\cline { 4 - 5 } Air conditioning & 50 & 50 & 0.9831 & 0.9976 \\
\hline Electric kettle & 50 & 50 & 0.9876 & 0.9912 \\
\hline
\end{tabular}

During the experiment, we also found that the efficiency of particle filter is mainly determined by the number of particles used.With the increase of the number of particles, efficiency is higher.But in this paper,the particle filter algorithm is not improved,which is the need to improve this article.

\section{Summary}

This paper mainly introduces the family load monitoring based on auxiliary particle filter algorithm, the process of state estimation using auxiliary particle filter algorithm is introduced in detail. And through this algorithm, the household electric load of one day was synthesized. And using MATLAB simulation, obtained five kinds of electrical transient waveforms, more accurately determine the electrical switch state. It illustrates the nonlinear state estimation method, which can be approximated by particle filtering, the algorithm is suitable for household load monitoring. And it is found that the efficiency of particle filter is mainly determined by the number of particles. With the increase of the number of particles, efficiency is higher. But in this paper, the auxiliary particle 
filter algorithm is not improved, which is the need to improve this article.

\section{Acknowledgements}

This work was financially supported by International S\&T Cooperation Program of China (2015 DFR10490).

\section{References}

[1] Hart G W.Nonintrusive appliance load monitoring[J].Proceedings of the IEEE,1992,80(12):1870-1891.

[2] Froehlich J,Larson E,Gupta S,et al.Disaggregated end-use energy sensing for the smart grid[J].IEEE Pervasive Computing,2010,10(1):28-39.

[3] Ridi A,Gisler C,Hennebert J.A Survey on Intrusive Load Monitoring for Appliance Recognition[C]//Pattern Recognition(ICPR),2014 22nd International Conference on.Stockholm:IEEE,2014:3702-3707.

[4] Gordon N,Salmond D.Novel approach to non-linear and no n-Gaussian Bayesian state estimation[J].Proc of Institute Electric Engineering,1993,140(2):107-113.

[5] F. Dellaert, D. Fox, W. Burgd, and S. Thrun. Monte carlo localization for mobile robots. In proc. IEEE International Conference on Robotics and Automation, Detroit, Mechigan, May, 1999.

[6] M. K. Pitt and N. Shephard. Filtering via simulation: Auxiliary particle filters. Journal of American Statistical Association,94(446): 590-599, June 1999.

[7] A. Doucet, N. de freitas, and N. Gordon. Sequential Monte Carlo methods in practice, Springer-verlag,2001.

[8] M. Isard and A. Blake. Condesation-conditional density propagation for visual tracking. Int. j. Comp. Vision, vol.29, no.1, pp:5-28, 1998.

[9] Handschin J E.Monte Carlo techniques for prediction and filtering of non-linear stochastic processes [J] .Automatica, 1970, 6(3): 555-563.

[10] L. Rabiner, "A tutorial on hidden Markov models and selected applications in speech recognition," Proc. IEEE, vol. 77, no. 2, pp. 257-286,Feb. 1989.

[11] M. S. Arulampalam, S. Maskell, N. Gordon, and T. Clapp, "A tutorial on particle filters for online nonlinear/non-Gaussian Bayesian tracking,” IEEE Trans. Signal Process., vol. 50, no. 2, pp. 174-188,Feb. 2002. 